\title{
Quel rôle pour les scénarios Facteur 4 dans la construction de la décision publique?
}

Sandrine Mathy, Meike Fink et Ruben Bibas

\section{(2) OpenEdition}

Journals

Édition électronique

URL : http://journals.openedition.org/developpementdurable/8802

DOI : $10.4000 /$ developpementdurable.8802

ISSN : 1772-9971

Éditeur

Association DD\&T

Référence électronique

Sandrine Mathy, Meike Fink et Ruben Bibas, «Quel rôle pour les scénarios Facteur 4 dans la construction de la décision publique? », Développement durable et territoires [En ligne], Vol. 2, n 1 | Mars 2011, mis en ligne le 28 février 2011, consulté le 19 avril 2019. URL : http://

journals.openedition.org/developpementdurable/8802 ; DOI : 10.4000/developpementdurable.8802

Ce document a été généré automatiquement le 19 avril 2019.

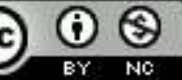

Développement Durable et Territoires est mis à disposition selon les termes de la licence Creative Commons Attribution - Pas d'Utilisation Commerciale 4.0 International. 


\title{
Quel rôle pour les scénarios Facteur 4 dans la construction de la décision publique?
}

\author{
Sandrine Mathy, Meike Fink et Ruben Bibas
}

1 L'émergence du dossier climatique et des impératifs de réduction des émissions de gaz à effet de serre (GES) a conduit à l'adoption en France en 2005 dans la loi de Programmation et d'Orientation de la Politique Energétique d'un objectif de division par 4 des émissions de GES ${ }^{1}$ d'ici 2050. Un certain nombre d'exercices de modélisation ont été élaborés dans le but d'éclairer la décision sur les modalités de réalisation de ces réductions d'émissions. Ces exercices représentent l'évolution du mix énergétique et ne couvrent que les émissions de $\mathrm{CO}_{2}$ issues de la combustion des énergies fossiles, soit au regard de la place privilégiée de l'agriculture dans notre pays, uniquement les $3 / 4$ de nos émissions ${ }^{2}$.

2 Cet article analyse les apports de ces exercices et clarifie leur rôle dans la construction de la décision publique. Nous revenons en détail dans la première partie sur les méthodes de construction de chacun de ces scénarios. Nous analysons leurs résultats dans la seconde partie. La disparité des résultats et les limites méthodologiques nous conduisent à nous interroger sur le rôle que devrait jouer la scénarisation dans la troisième partie. L'enjeu serait d'accompagner les travaux de modélisation par des échanges au sein d'une plateforme entre modélisateurs, économistes, technologues, sociologues et représentants de la société civile. Pour renforcer leur légitimité, les modèles analysant le Facteur 4 et les scénarios qui en découlent doivent ainsi participer à l'appropriation sociale des résultats scientifiques.

\section{Scénarios Facteur 4: approches et méthodologie}

\subsection{Présentation des scénarios}

Sept exercices ont été identifiés au niveau français. 
4 Exercice 1. La division par 4 des émissions de dioxyde de carbone en France d'ici 2050 (MIES - 2004). Cet exercice mené par Pierre Radanne - ancien président de l'ADEME, à l'époque membre de la Mission Interministérielle sur l'Effet de Serre (MIES) - et rendu à la MIES en 2004 présente des images en 2050 du paysage énergétique français selon 5 variantes d'offre technologique s'appuyant toutes sur des niveaux équivalents de projection de la demande d'énergie finale en 2050 : MIES-Nuke (développement accru du nucléaire + pénétration de l'électricité dans tous les usages y compris le transport) ; MIES$\mathrm{H}$ (développement d'une filière hydrogène alimentée par des centrales nucléaires) ; MIESRogo (équilibre entre le recours au nucléaire et le développement de la cogénération et des renouvelables) ; MIES-Séquest (développement de la séquestration du $\mathrm{CO}_{2}$; $\mathrm{MIES}-\mathrm{sN}+$ (sortie du nucléaire avec séquestration $\mathrm{du} \mathrm{CO}_{2}$ ).

5 Exercice 2. Politique énergétique nationale et lutte contre l'effet de serre (Prévot 2007 (Développé dans Prévot 2004 et repris dans Prévot 2007). Henri Prévot, Ingénieur des Mines, a produit un scénario à l'horizon 2030 reposant sur: une stabilisation de la demande d'énergie finale et un recours massif aux agrocarburants et aux véhicules électriques couplés à un déploiement nucléaire important.

6 Exercice 3. Etude pour une prospective énergétique concernant la France (Observatoire de l'Energie, Direction Générale de l'Energie et des Matières Premières ENERDATA, LEPII - 2005). La particularité de ce scénario, issu d'une commande ministérielle, est que les émissions continuent à croître jusqu'en 2030 puis décroissent d'autant plus fortement par la suite. Ce scénario s'appuie sur le modèle Med-Pro couplé à POLES.

7 Exercice 4. Scénario négaWatt 2006, pour un avenir énergétique sobre, efficace et renouvelable (négaWatt - 2006). L'association négaWatt regroupant plus d'une centaine d'experts techniques de l'efficacité énergétique et des énergies renouvelables a élaboré en 2003 puis 2006 un scénario énergétique avec non-renouvellement des centrales nucléaires. Cet exercice se base sur les usages énergétiques que sont la chaleur, la mobilité et l'électricité. L'approche adoptée s'organise autour du triptyque : i) sobriété énergétique, ii) efficacité énergétique, iii) déploiement des énergies renouvelables (ENR).

8 Exercice 5. NégaTep - 2007 (Acket 2007). L'association Sauvons le Climat est constituée de retraités pour beaucoup ayant œuvré dans le domaine du nucléaire. Cette association a développé le scénario négaTep qui met en avant selon leur manifeste "à la fois des économies d'énergie et le développement de l'énergie nucléaire et des énergies renouvelables ».

Exercice 7. Perspectives énergétiques de la France à l'horizon 2020-2050 (Centre d'Analyse Stratégique, Commission énergie - Jean Syrota - 200733). Le premier ministre avait demandé au Centre d'Analyse Stratégique de réunir une commission de haut niveau en vue de " dégager les principales orientations opérationnelles et préconisations de politique publique en matière de maîtrise de la demande énergétique, de transports et d'aménagement, d'offre d'énergies et de régulation du marché énergétique ». Cette commission présidée par Jean Syrota s'est appuyée sur 2 expertises extérieures en matière de modélisation: le modèle Med-Pro couplé à POLES et le modèle Markal-Times.

10 Exercice 8. D'autres exercices de quantification de réduction des émissions de $\mathrm{CO}_{2}$ existent mais ne seront pas ici étudiés, et ce pour différentes raisons. Le scénario réalisé par l'association Virage Energie représentant le Facteur 4 se situe à l'échelle d'un territoire, en l'occurrence la région Nord-Pas de Calais ${ }^{4}$. Un exercice de quantification a été mené par l'équipe d'Enerdata, relatif à la mise en œuvre des objectifs du Grenelle de 
l'Environnement, mais il ne se place pas dans la perspective d'un facteur 4 en 2050. Un exercice de quantification de la valeur carbone (Quinet 2009). Enfin, la Commission de Boissieu (2006) sur le Facteur 4 avait également établi sur la base d'une revue de la littérature une liste de recommandations mais celles-ci n'avaient donné lieu à aucune quantification.

\subsection{Description des méthodologies}

11 Les scénarios énergétiques évoqués ici soit représentent la trajectoire entre une année de référence et 2050, soit se contentent de donner l'image du mix énergétique à l'horizon souhaité pour répondre à une demande d'énergie sous contrainte carbone. Pour cela, les modèles ou exercices dits d'ingénieur ou bottom-up ${ }^{5} s^{\prime}$ appuient sur des projections de la demande d'énergie finale ${ }^{6}$ correspondant le plus souvent à des hypothèses de croissance économique et démographique prédéterminées. Selon des hypothèses techniques plus ou moins détaillées sur l'évolution de la performance des technologies, sur les potentiels des énergies renouvelables... et selon une méthodologie spécifique à chaque exercice, un mix technologique et énergétique est calculé déterminant la part respective de chacune des technologies et formes d'énergie dans l'énergie finale consommée par secteur et dans l'énergie primaire correspondante. La méthodologie de calcul peut être (1.1. Présentation des scénarios) soit un tableur relevant plus de l'analyse de données, empilant, à partir d'hypothèses sur l'évolution de la demande d'énergie finale, des potentiels technologiques (négaWatt, Prévot, négaTep); soit une optimisation du mix énergétique avec force détails technologiques : Markal (utilisé dans Syrota-Markal) minimise le coût total actualisé en investissement et fonctionnement des technologies pour répondre à une demande en biens et services exogène ; soit une simulation technicoéconomique qui établit l'équilibre du marché de l'énergie entre offre et demande en fonction d'un prix de l'énergie qui peut être endogène (Enerdata et Syrota-MedPro) : côté demande, le modèle Médée (modèle Top-Down) évalue la demande finale d'énergie avec un fort niveau de désagrégation au niveau national à l'horizon 2030 ou 2050 ; et côté offre, le modèle POLES (modèle Bottom-Up) est un modèle mondial régionalisé d'équilibre partiel du système énergétique. La résolution de l'équilibre offre/demande débouche sur un prix de l'énergie et sur un mix énergétique chaque année jusqu'en 2050 pour répondre à cette demande selon des données de coûts d'investissement et de fonctionnement des technologies intégrant ce prix de l'énergie et éventuellement un prix du carbone (voir plus bas). Les modèles d'optimisation ou de simulation utilisés dans les exercices étudiés ici (et $a$ fortiori les tableurs) ne tiennent pas compte des rétroactions et interactions entre l'évolution du système énergétique et les dynamiques macroéconomiques (dans le meilleur des cas, POLES ne fait qu'intégrer un prix de l'énergie endogène) comme le font les modèles d'équilibre général qui représentent l'impact des politiques climatiques sur les prix des différents biens et services, sur l'emploi, sur les niveaux de croissance... Les hypothèses de cadrage macroéconomique sont donc exogènes. 
Tableau 1 : Types d'approche des différents exercices de représentation des réductions d'émissions de $\mathrm{CO}_{2}$ en France

\begin{tabular}{|c|c|c|c|c|c|c|c|}
\hline & $\begin{array}{c}\text { négaWatt } \\
\mathbf{2 0 0 6}\end{array}$ & $\begin{array}{c}\text { négaTep } \\
\mathbf{2 0 0 7}\end{array}$ & $\begin{array}{c}\text { Enerdata } \\
\mathbf{2 0 0 5}\end{array}$ & $\begin{array}{c}\text { Prévot } \\
\mathbf{2 0 0 7}\end{array}$ & $\begin{array}{c}\text { Mies } \\
\mathbf{2 0 0 4}\end{array}$ & \multicolumn{2}{c|}{$\begin{array}{c}\text { Syrota } \\
\mathbf{2 0 0 7}\end{array}$} \\
\hline $\begin{array}{c}\text { Type de } \\
\text { modélisation }\end{array}$ & Bottom-up & Bottom-up & $\begin{array}{c}\text { Bottom-up } \\
+ \text { Top Down }\end{array}$ & Bottom-up & Bottom-up & $\begin{array}{c}\text { Bottom-up } \\
+ \text { Top Down }\end{array}$ & Bottom-up \\
\hline $\begin{array}{c}\text { Type } \\
\text { d'exercice }\end{array}$ & Simulation & Simulation & Simulation & Simulation & Simulation & Simulation & Optimisation \\
\hline Modèle & $\begin{array}{c}\text { Analyse de } \\
\text { données }\end{array}$ & $\begin{array}{c}\text { Analyse de } \\
\text { données }\end{array}$ & $\begin{array}{c}\text { MEDEE } \\
\text { +POLES }\end{array}$ & $\begin{array}{c}\text { Analyse de } \\
\text { données }\end{array}$ & $\begin{array}{c}\text { Analyse de } \\
\text { données }\end{array}$ & MEDEE+POLES & $\begin{array}{c}\text { MARKAL- } \\
\text { TIMES }\end{array}$ \\
\hline
\end{tabular}

\subsection{Hypothèses de cadrage macroéconomique}

Les hypothèses de cadrage macroéconomique servant à l'évaluation de la demande renvoient aux hypothèses sur la démographie et sur la croissance économique (Tableau 1: Types d'approche des différents exercices de représentation des réductions d'émissions de $\mathrm{CO} 2$ en France). Entre 2003 et 2006, l'INSEE a revu à la hausse ses projections démographiques avec un différentiel de 6 millions de personnes en 2050, ce qui entraîne donc toutes choses égales par ailleurs une augmentation de $10 \%$ environ des besoins énergétiques. Les hypothèses de croissance économique apparaissent contrastées, surtout lorsqu'elles sont croisées avec les hypothèses démographiques : dans le scénario Enerdata, la croissance du revenu par habitant est de $137 \%$ en 2050, soit un taux de croissance annuel moyen du revenu per capita de 2,18\%. Comparativement, sur les 30 dernières années, la croissance économique a été de 2,06\% et la croissance du revenu par habitant de 1,56\%. Hormis les scénarios Enerdata et Prévot, les autres scénarios sont donc plus en ligne avec les tendances passées. A noter que le scénario négaWatt ne s'appuie pas sur une trajectoire de croissance économique car ce critère est rejeté comme allant à l'encontre du concept de sobriété, mais sur une évaluation des besoins fondamentaux de chaleur, de mobilité et d'électricité spécifique.

Tableau 2 : Hypothèses démographique et économique des scénarios

\begin{tabular}{|c|c|c|c|c|}
\hline & $\begin{array}{c}\text { Scénario } \\
\text { démographique }\end{array}$ & tcam économique & $\begin{array}{c}\text { Croissance revenu } \\
\text { par habitant }\end{array}$ & $\begin{array}{c}\text { Revenu par habitant } \\
\text { en } 2050 \text { (base } \\
100=2000 \text { ) }\end{array}$ \\
\hline Prévot & 64 millions en 2030 & $2,3 \%$ & $2,13 \%$ & $149($ en 2030$)$ \\
\hline négaWatt & \multirow{3}{*}{$\begin{array}{c}\text { INSEE } 2003 \\
64 \text { millions en } 2050\end{array}$} & - & - & - \\
\hline MIES & & $1,7 \%$ & $1,58 \%$ & 187 \\
\hline Enerdata & & $2,3 \%$ & $2,18 \%$ & 237 \\
\hline NégaTep & 65 millions en 2050 & - & - & - \\
\hline Markal & \multirow{2}{*}{$\begin{array}{c}\text { INSEE } 2006 \\
67 \text { millions en } 2030 \\
70 \text { millions en } 2050\end{array}$} & $1,74 \%$ & $1,50 \%$ & 181 \\
\hline Med-Pro & & $1,74 \%$ & $1,50 \%$ & 181 \\
\hline
\end{tabular}

13 Les hypothèses de croissance économique et démographique permettent la projection de la demande de biens et services. En cas de prise en compte des rétroactions sur l'économie, elles servent également à l'évaluation des politiques menées sur les niveaux d'activités au sein d'un secteur ou à la mise en exergue de possibles effets d'évictions des investissements nécessaires sur d'autres secteurs. Alors que le cheminement vers une division par 4 des émissions de GES ne pourra se faire qu'avec une profonde refonte du contenu de la croissance et des consommations du fait des rétroactions sur l'économie 
des politiques correspondantes, celles-ci ne sont pas prises en compte dans les différents scénarios analysés.

\subsection{Prix de l'énergie et prix du carbone}

De la même manière, trajectoires d'émissions de $\mathrm{CO}_{2}$, consommations d'énergie et choix technologiques en France sont indissociables des perspectives d'évolutions du prix de l'énergie carbonée au sens large (prix de la ressource + prix du carbone), lui-même indissociable des tensions sur les ressources en énergies fossiles au niveau mondial et donc in fine des hypothèses à la fois sur les réserves fossiles et sur les politiques climatiques et énergétiques au niveau mondial. Deux options se présentent : 1) Réaliser un facteur 4 en France dans un contexte mondial de forte tension sur les ressources fossiles en diminuera le coût supplémentaire car le prix des énergies fossiles sera d'ores et déjà élevé ; les énergies non carbonées se développeront plus facilement. Le prix du carbone nécessaire à l'atteinte de l'objectif sera moindre. 2) Réaliser un facteur 4 en France dans un contexte mondial de lutte active contre le réchauffement climatique qui repoussera dans le temps le peak-oil et l'augmentation des prix du pétrole, nécessitera un prix du carbone plus important.

Dans les exercices relevant d'analyse de données, les prix de l'énergie et du carbone ne sont pas pris en compte pour la résolution du mix énergétique en 2050. Dans le meilleur des cas, une discussion est juste menée sur un prix du carbone apte à induire les basculements technologiques décrits. Pour Prévot ${ }^{7}$, le prix du baril de pétrole ne pourra pas dépasser le coût de production de carburant liquide à partir de charbon ${ }^{8}$ qu'il estime inférieur à $50 \$ / b l$. Dans ces conditions, le prix du carbone nécessaire à la réalisation du Facteur 4 correspond au niveau permettant la pénétration des technologies non carbonées et la stabilisation des consommations. Pour conserver la compétitivité industrielle, l'auteur considère que ce prix du carbone ne devra pas s'appliquer aux industries et au transport de marchandises. Pour les scénarios de la MIES, une hausse de la valeur du carbone serait sans effet dommageable sur l'économie si elle était compensée par une baisse dans les mêmes proportions de la dépense énergétique. Le prix du carbone est alors défini par la valeur permettant de maintenir constantes en valeur ou en part du PIB les dépenses énergétiques de la France par rapport à un scénario de référence sans économie d'énergie. Cette valeur va donc dépendre de l'hypothèse sur l'évolution du prix de l'énergie. Deux hypothèses sont discutées: une stabilisation du prix du baril aux niveaux de 2000 et un doublement.

Dans les scénarios issus de modèles, le prix du carbone est une variable endogène ajoutée au prix de l'énergie pour former le prix aux consommateurs. Le prix de l'énergie est fixé de manière exogène dans un exercice sur la France mais ce prix est censé refléter un équilibre sur les marchés mondiaux de l'énergie. Les hypothèses utilisées dans l'exercice Enerdata et dans l'exercice du CAS sont fortement contrastées. Selon Enerdata, le prix de l'énergie utilisé est issu de résultats de simulation du modèle mondial. Ainsi, le scénario de référence pour la France se place dans un contexte mondial sans politiques climatiques. De ce fait, les tensions sur la ressource apparaissent et le prix du baril atteint $50 € / b l$ en 2050. Dans le scénario Facteur 4 pour la France, l'équilibre offre/demande sur les marchés de l'énergie se déroule dans un contexte de convergence mondiale des émissions par tête à long terme. La demande de pétrole diminue donc fortement et le prix du pétrole chute en dessous de 20\$/bl en 2050. Dans les scénarios du CAS (Syrota-Markal 
et Syrota MedPro), la commission Énergie en charge de cadrer les grandes lignes des scénarios a fait l'hypothèse d'un prix de l'énergie élevé et cyclique : entre 50 et $80 \$ /$ baril jusqu'en 2015, entre 100 et $150 \$ /$ baril de 2015 à 2030 et $100 \$ /$ baril au-delà en ligne avec les prévisions en 2007 du Conseil Mondial de l'Énergie.

Pour les modèles POLES (utilisé pour les scénarios Enerdata et Syrota-MedPro) et Markal (Syrota-Markal), le prix du carbone apparaît comme la valeur duale (c'est-à-dire la valeur implicite) de la contrainte carbone. Il correspondrait au prix du carbone à appliquer au niveau de l'économie si aucune autre mesure n'était mise en œuvre, puisque les décisions orientant les déploiements technologiques sont dépendantes de bien d'autres variables que le seul signal prix. C'est la valeur nécessaire dans les modèles pour faire pénétrer les technologies permettant de répondre à l'objectif de réduction. Or, il apparaît que les modèles POLES et Markal utilisés en l'état (avec les technologies et leurs hypothèses et avec les niveaux de demande introduits au préalable) butent rapidement sur des niveaux de réduction des émissions trop faibles. Ainsi, dans le scénario Enerdata, le Facteur 3 n'est atteint qu'en introduisant de nouvelles technologies bas carbone et Syrota-Markal est obligé d'aller chercher des prix du carbone de $30 \mathrm{k} € / \mathrm{tC}$ pour faire pénétrer dans le mix énergétique des technologies non carbonées et réaliser effectivement du Facteur 4. Ce résultat n'étant pas recevable, l'exercice final réalisé avec Markal s'est limité à des niveaux de réductions moindres mais avec un prix du carbone restant dans le domaine de l'acceptable.

18 Ceci est révélateur de l'incapacité de ces modèles à représenter des bifurcations au niveau de la demande d'énergie. Avec de tels niveaux de réductions d'émission et de tels prix du carbone, il est peu envisageable de pouvoir se contenter de raisonner à demande exogène fixée pour Syrota-Markal ou avec une demande évoluant selon une élasticité prix de la demande calibrée sur le passé pour Enerdata et Syrota-Med-Pro alors que les demandes vont forcément connaître de fortes bifurcations et réorientations dans un contexte de Facteur 4 .

\section{Analyse comparative et critique des résultats des scénarios F4}

\subsection{Un résultat non atteint et limité méthodologiquement}

19 Un seul scénario existant aboutit effectivement à un facteur 4 (2. Analyse comparative et critique des résultats des scénarios $\mathrm{F} 4$ ). C'est le scénario négaWatt avec une division par 4,2 des émissions de $\mathrm{CO}_{2}$. Les autres scénarios atteignent au mieux un facteur 3 mis à part les scénarios MIES avec un facteur 3,4. Il est à noter que le modèle Syrota-Markal peine " au-delà de ce facteur de réduction à trouver des solutions de substitution utilisables pour réduire davantage les émissions de $\mathrm{CO}_{2}$ " (Rapport Syrota, 2007). Une valeur du carbone de $30 \mathrm{k} € / \mathrm{tC}$ serait nécessaire pour atteindre un facteur 4. Le scénario SyrotaMedPro ne pourrait atteindre quant à lui un facteur 3,8 (scénario non retenu) que grâce au recours à la production d'électricité par des centrales à charbon associées à de la séquestration géologique à hauteur de $60 \mathrm{MtCO}_{2}$ en 2050 . Hormis cela, les réductions obtenues sont celles mentionnées dans le tableau ci-dessous. 
Tableau 3 : Récapitulatif des objectifs de réduction dans les différents exercices par rapport aux émissions de $\mathrm{CO}_{2}$ seul en 2000

\begin{tabular}{|c|c|c|c|c|c|c|c|}
\hline & $\begin{array}{c}\text { négaWatt } \\
\mathbf{2 0 0 6}\end{array}$ & $\begin{array}{c}\text { négaTep } \\
\mathbf{2 0 0 7}\end{array}$ & $\begin{array}{c}\text { Enerdata } \\
\mathbf{2 0 0 5}\end{array}$ & $\begin{array}{c}\text { Prévot } \\
\mathbf{2 0 0 7}\end{array}$ & $\begin{array}{c}\text { Mies } \\
\mathbf{2 0 0 4}\end{array}$ & $\begin{array}{c}\text { Syrota- } \\
\text { Med-Pro } \\
\mathbf{2 0 0 7}\end{array}$ & $\begin{array}{c}\text { Syrota - } \\
\text { Markal } \\
\mathbf{2 0 0 7}\end{array}$ \\
\hline $\begin{array}{c}\text { Objectif de } \\
\text { réduction }\end{array}$ & 4,2 & 3,1 & 2,9 & 2,9 & 3,4 & 3 & 2,9 \\
\hline
\end{tabular}

De plus, des considérations méthodologiques limitent encore la portée de ces résultats. Tout d'abord, seul le $\mathrm{CO}_{2}$ issu des émissions énergétiques est pris en compte dans ces exercices, soit $75 \%$ des émissions françaises. D'autre part, les émissions de $\mathrm{CO}_{2}$ prises en compte sont par convention les émissions émises sur le territoire : elles comptabilisent les émissions produites pour les biens exportés, mais pas celles des biens importés. Selon Nakano et al. (2009), si l'on comptabilise les émissions de la France sur la base de la consommation domestique (donc en incluant les importations et en excluant les exportations) et non sur la base de la production domestique, les émissions françaises seraient $30 \%$ supérieures. Aucun des exercices ne prend en compte cette externalisation des émissions alors même qu'une forte contrainte carbone en France pourrait conduire à reporter une partie de la consommation vers l'importation de biens de pays à contrainte carbone moindre.

21 Au regard de ces éléments, aucun des exercices existant ne permet de réaliser le Facteur 4 sur l'ensemble des émissions «consommées » par les Français. Comment doit-être compris ce résultat? Comme un révélateur de l'impossibilité à atteindre cet objectif ? Comme la preuve que les modèles ne sont pas adaptés à cette évaluation? Cette impasse méthodologique est d'autant plus surprenante que des scénarios européens issus de commande gouvernementale représentent des évolutions énergétiques bien plus en rupture qu'un facteur 4 en France: par exemple, le scénario du ministère de l'écologie allemand suppose une réduction du $\mathrm{CO}_{2}$ de 85\% en 2050 par rapport à 1990 (Nitsch, 2008) et le scénario «ZeroCarbonBritain » (Allen 2007) présente une économie britannique sans aucune énergie fossile en 2027.

\subsection{Quelles réductions dans quels secteurs?}

Avant de détailler les réductions d'émissions mises en œuvre dans chacun des scénarios, le panorama en 2000 que nous prenons comme année de référence pour la suite de l'analyse est le suivant :

Tableau 4 : Emissions sectorielles de $\mathrm{CO}_{2}$ et de $\mathrm{CO}_{2}$ eq en 2000 et évolutions depuis 1990 (en Mt)

\begin{tabular}{|c|c|c|c|c|c|c|}
\hline & Energie & Industrie & $\begin{array}{c}\text { Résidentiel/ } \\
\text { Tertiaire }\end{array}$ & $\begin{array}{c}\text { Agriculture/ } \\
\text { Sylviculture }\end{array}$ & Transports & Total hors UCTF \\
\hline Emissions $\mathbf{C O}_{2}$ & 65 & 104 & 88 & 8,6 & 133,4 & 398 \\
\hline Emissions $\mathbf{C O}_{2}$ eq & 71 & 133 & 96 & 112 & 136,5 & 548 \\
\hline Evolution $\mathrm{CO}_{2} \mathbf{1 9 9 0}-\mathbf{2 0 0 0}$ & $-5,8 \%$ & $-8 \%$ & $+6 \%$ & $-8,5 \%$ & $+14 \%$ & $-1,8 \%$ \\
\hline Evolution $\mathbf{C O}_{2}$ eq $\mathbf{1 9 9 0}-\mathbf{2 0 0 0}$ & $-7,8 \%$ & $-15,3 \%$ & $+7,9 \%$ & $-3,4 \%$ & $+16,1 \%$ & $-1,4 \%$ \\
\hline
\end{tabular}

Note : UCTF : utilisation des terres, leur changement et la forêt

Pour atteindre un facteur 4 entre 2000 et 2050, alors que les émissions de $\mathrm{CO}_{2}$ seul étaient de $398 \mathrm{MtCO}_{2}$ et que l'on considère des facteurs de réduction identiques sur l'ensemble 
des GES, il faudrait donc atteindre $98 \mathrm{MtCO}_{2}$ et réduire les émissions de $300 \mathrm{MtCO}_{2}$ en 2050 par rapport aux émissons de 2000. Le Tableau 4 : Emissions sectorielles de $\mathrm{CO} 2$ et de $\mathrm{CO} 2$ eq en 2000 et évolutions depuis 1990 (en Mt) montre les réductions sectorielles par rapport à 2000 obtenues dans chacun des scénarios.

Tableau 5 : Répartition sectorielle des réductions d'émissions $\left(\mathrm{MtCO}_{2}\right)$ par rapport aux émissions sectorielles de 2000 et niveau de réduction total des différents scénarios en 2050

\begin{tabular}{|c|c|c|c|c|c|}
\hline & Transports & $\begin{array}{c}\text { Résidentiel/ Tertiaire/ } \\
\text { Agriculture }\end{array}$ & Industrie & CcS $^{*}$ & $\begin{array}{c}\text { Réductions } \\
\text { totales }\end{array}$ \\
\hline negaWatt & 109 & 113 & 70 & - & 292 \\
\hline MIES - F4 RcogN & 125 & 84 & 60 & - & 269 \\
\hline MIES - F4 Séquest & 86 & 67 & -7 & 123 & 269 \\
\hline MIES - F4 Nuke & 128 & 88 & 52 & - & 268 \\
\hline MIES - F4 sN+S & 91 & 16 & -40 & 200 & 267 \\
\hline MIES - F4 H2 & 105 & 106 & 55 & - & 266 \\
\hline negaTep & 99 & 105 & 51 & - & 255 \\
\hline Enerdata & 82 & 121 & 47 & - & 249 \\
\hline Prévot (2030) & 111 & 94 & 43 & - & 247 \\
\hline Syrota-MEDPRO & 106 & 55 & 62 & - & 223 \\
\hline Syrota-MARKAL & 85 & 83 & 31 & - & 199 \\
\hline Moyenne & 101 & 85 & 39 & & \\
\hline Ecart type & 16 & 30 & 33 & & \\
\hline
\end{tabular}

[note* : Le captage et le stockage du $\mathrm{CO}_{2}$ (CCS) est un procédé technique qui sépare le dioxyde de carbone des gaz produits par les grandes centrales thermiques, comprime le $\mathrm{CO}_{2}$, puis le transporte dans un endroit où il peut être conservé dans des formations géologiques, dans des anciens puits de pétrole ou dans l'océan. Cette technologie pose de nombreux problèmes. Elle entrainerait des surcoûts de production d'électricité importants et des consommations d'énergie supplémentaires. La question de la pérennité du stockage se pose ainsi que celle des risques environnementauxfonctions des réservoirs géologiques retenus - et sanitaires.]

Le secteur des transports, habituellement considéré comme le plus délicat pour mettre en œuvre des réductions, contribue en moyenne le plus aux réductions d'émissions (car il est le principal secteur émetteur). Ces réductions sont obtenues par des mesures de maitrise de la mobilité par l'aménagement du territoire, de développement des modes de transports alternatifs à la route (transport ferroviaire, transports collectifs urbains), de gains en efficacité énergétique des véhicules routiers, de substitution vers des technologies électriques ou hybrides et enfin par la percée des agrocarburants, dont les émissions pour la production et la culture ne sont jamais prises en compte. Les conséquences sur le secteur et les marchés agricoles sont, elles aussi, négligées.

Le secteur résidentiel/tertiaire est le second contributeur en moyenne avec une variabilité des niveaux de réduction très forte. Ceci peut paraître étonnant car, pour ce qui est du bâtiment, il y a une forte convergence sur les mesures à mettre en œuvre : renforcement des réglementations thermiques et rénovation du parc bâti. Ceci s'explique par des appréciations très différentes de la part du gisement d'économies d'énergie qui peut être exploitée, des perspectives de déploiement de technologies très efficaces telles que les réseaux de chaleur, la cogénération ou les chauffe-eaux solaires ou bien des perspectives de réduction des consommations d'électricité spécifiques.

Le secteur de l'industrie est le dernier secteur contributeur en moyenne avec un écarttype à peu près égal à la moyenne de sa contribution (i.e. les incertitudes sont énormes). Il est à noter également le niveau extrèmement élevé de la contribution de la CCS dès lors que cette technologie est considérée comme disponible ${ }^{9}$. 
Dans la suite, nous passons les évolutions sectorielles en revue dans chacun des scénarios. Ceci permet d'analyser pour chacun des secteurs à la fois l'évolution de la demande d'énergie finale et du mix énergétique, ainsi que la répartition sectorielle des efforts de réduction au niveau global. Pour cela, nous comparerons à chaque fois les réductions d'émissions par rapport au niveau observé en 2000 et nous menons une décomposition à la Kaya selon l'évolution de la population, l'évolution de la demande d'énergie finale par habitant et le contenu en carbone de l'énergie finale :

$$
\begin{aligned}
& E_{\text {sect }}^{\circ}(2050)=P O P(2050) \underbrace{\frac{E F_{\text {sect }}(2050)}{P O P(2050)}}_{\begin{array}{c}
\text { Consommation } \\
\text { d'énergie finale } \\
\text { sectorielle par } \\
\text { habitant }
\end{array}} \cdot \underbrace{\frac{E_{\text {sect }}^{\circ}(2050)}{E F_{\text {sect }}(2050)}}_{\begin{array}{c}
\text { Contenu en } \\
\text { carbone de l'énergie } \\
\text { finale sectorielle }
\end{array}} \\
& \log \frac{E_{\text {sect }}^{\circ}(2050)}{E_{\text {sect }}^{\circ}(2000)}=\log \frac{P O P(2050)}{P O P(2000)}+\log \frac{\frac{E F_{\text {sect }}(2050)}{P O P(2050)}}{\frac{E F_{\text {sect }}(2000)}{P O P(2000)}}+\log \frac{\frac{E_{\text {sect }}^{\circ}(2050)}{E F_{\text {sect }}(2050)}}{\frac{E_{\text {sect }}^{\circ}(2000)}{E F_{\text {sect }}(2000)}}
\end{aligned}
$$

29 Ceci nous permet de gommer l'impact sur les émissions des hypothèses différentes d'évolution démographique et de séparer la contribution aux réductions d'émissions de la substitution énergétique ou technologique (capturée dans le coefficient d'évolution du contenu carbone de l'énergie finale) et de la diminution de la demande finale (capturée dans le coefficient d'évolution de la demande d'énergie finale sectorielle par habitant, qui peut traduire soit des comportements plus sobres, soit des gains en termes d'efficacité énergétique). Les résultats sont visibles dans la Figure 1 qui montre en haut cette décomposition et en bas les facteurs de réduction réalisés pour chacun des secteurs et des scénarios.

Figure 1: Décomposition Kaya des réductions d'émissions par secteur pour tous les scénarios et facteur de réduction sectoriel par rapport à 2000
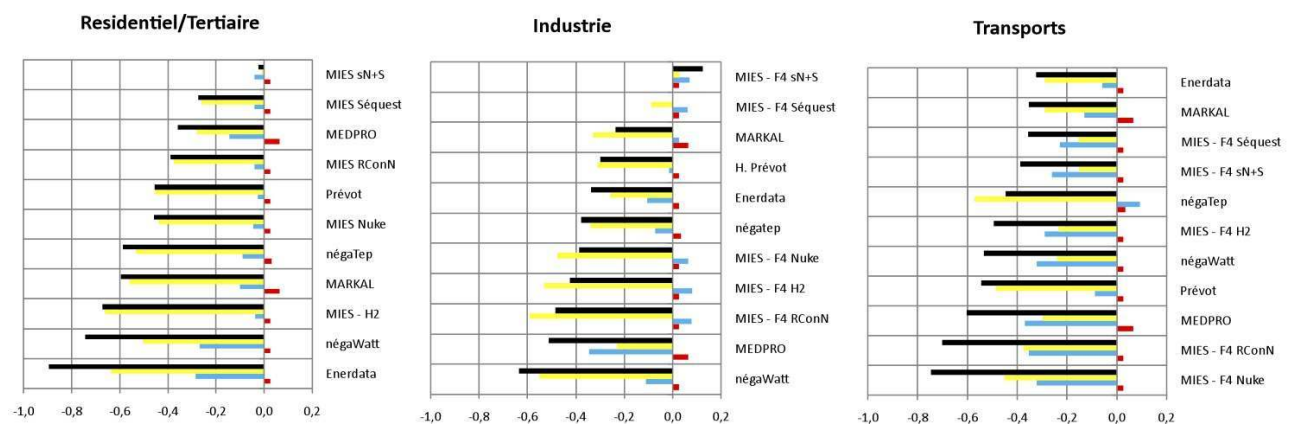


\begin{tabular}{|c|c|c|c|c|c|c|c|c|}
\hline & Habitat & $\begin{array}{c}\text { Facteur } \\
2050\end{array}$ & & Industrie & & & Transports & $\begin{array}{c}\text { Facteur } \\
2050\end{array}$ \\
\hline $2000 \mathrm{MtCO} 2$ & 121 & & $2000 \mathrm{MtCO} 2$ & 100 & & $2000 \mathrm{MtCO} 2$ & 161 & \\
\hline ENERDATA & 15 & 8,1 & $\mathrm{nW}$ & 23,3 & 4,3 & MIES Nuke & 28,3 & 5,7 \\
\hline $\mathrm{nW}$ & 21,8 & 5,6 & MEDPRO & 30,8 & 3,2 & $\begin{array}{c}\text { MIES } \\
\text { RCogN }\end{array}$ & 31,4 & 5,1 \\
\hline MIES H2 & 25,7 & 4,7 & $\begin{array}{c}\text { MIES } \\
\text { RCogN }\end{array}$ & 32,7 & 3,1 & MEDPRO & 40,2 & 4,0 \\
\hline MARKAL & 30,8 & 3,9 & MIES H2 & 37,7 & 2,7 & $\begin{array}{c}\text { Prévot - } \\
2030\end{array}$ & 45,8 & 3,5 \\
\hline négaTep & 31,6 & 3,8 & MIES Nuke & 41 & 2,4 & $\mathrm{nW}$ & 47 & 2,5 \\
\hline MIES Nuke & 42,2 & 2,9 & négaTep & 42 & 2,4 & MIES H2 & 51,4 & 3,1 \\
\hline $\begin{array}{c}\text { Prévot - } \\
2030\end{array}$ & 42,5 & 2,8 & ENERDATA & 46 & 2,2 & négaTep & 57,6 & 2,8 \\
\hline $\begin{array}{c}\text { MIES } \\
\text { RcogN }\end{array}$ & 49,4 & 2,5 & $\begin{array}{l}\text { Prévot - } \\
2030\end{array}$ & 50,4 & 2,0 & MIES sN+S & 64,8 & 2,5 \\
\hline MEDPRO & 53,0 & 2,3 & MARKAL & 58 & 1,7 & $\begin{array}{c}\text { MIES } \\
\text { Séquest }\end{array}$ & 69,6 & 2,3 \\
\hline $\begin{array}{c}\text { MIES } \\
\text { Séquest }\end{array}$ & 64,5 & 1,9 & $\begin{array}{c}\text { MIES } \\
\text { Séquest. }\end{array}$ & 100 & 1,0 & MARKAL & 71,3 & 2,3 \\
\hline MIES sN+S & 114,7 & 1,1 & MIES sN+S & 133,2 & 0,8 & ENERDATA & 76 & 2,1 \\
\hline
\end{tabular}

\section{Le secteur des transports}

30 Les scénarios conduisant aux réductions d'émissions les plus importantes dans le secteur des transports sont ceux couplant une forte réduction de la consommation d'énergie finale par habitant et une forte décarbonisation de l'énergie consommée. Ainsi, le scénario menant aux plus fortes réductions dans le secteur des transports est le scénario MIES-Nuke suivi par le scénario MIES-RcogN.

La contribution de la diminution du contenu carbone de l'énergie finale consommée dans les transports est très importante pour les quatre scénarios MIES-Nuke, MIES-RcogN, négaTep et Prévot. Dans les trois premiers ceci est dû à une très forte pénétration de l'électricité nucléaire dans le secteur des transports (Figure 2) et dans Prévot aux agrocarburants de deuxième génération $(51 \%)$ dont la production nécessite de grandes quantités d'électricité nucléaire et pour lesquels les émissions liées aux usages du sol ne sont pas comptabilisées. Il est également remarquable que le scénario avec développement de l'hydrogène MIES-H2 ne permet pas de faire baisser significativement le contenu en carbone de l'énergie consommée dans les transports.

Ces graphiques montrent que les résultats butent sur des visions différentes concernant les gains potentiels pour la réduction de la consommation d'énergie finale par habitant. Le scénario négaTep projette même une hausse de cette consommation. Dans les scénarios Syrota-MedPro, Enerdata et Syrota-Markal la baisse est très limitée du fait de la structure même des modèles qui ne permettent pas des inflexions notables de la demande d'énergie et de changement de comportement.

Au final, la plupart des scénarios décrivent une réduction de la demande d'énergie finale par habitant. Les moyens mis en œuvre pour y parvenir sont diversifiés : véhicules plus économes et moins émetteurs, transferts modaux (Enerdata multiplie le rail par 5,1, le fluvial par 3,5), rupture technologique concernant l'énergie utilisée (hydrogène dans MIES-H2, véhicules au gaz dans Syrota-Markal) ou baisse des km parcourus obtenus par une limitation des besoins de mobilité dépendant d'un véhicule individuel. Le scénario négaWatt prévoit quant à lui une légère augmentation des $\mathrm{km}$ parcourus par personne, couplée à une augmentation de l'utilisation des transports en commun, un recours aux agrocarburants et un taux de remplissage supérieur. Il est toutefois difficile d'évaluer la part de chacune de ces orientations dans les réductions d'émissions. 
Figure 2 : Part de l'électricité dans la consommation d'énergie finale dans les transports en 2050 dans les différents scénarios

\section{Part de l'électricité dans les transports (Prévot en 2030)}

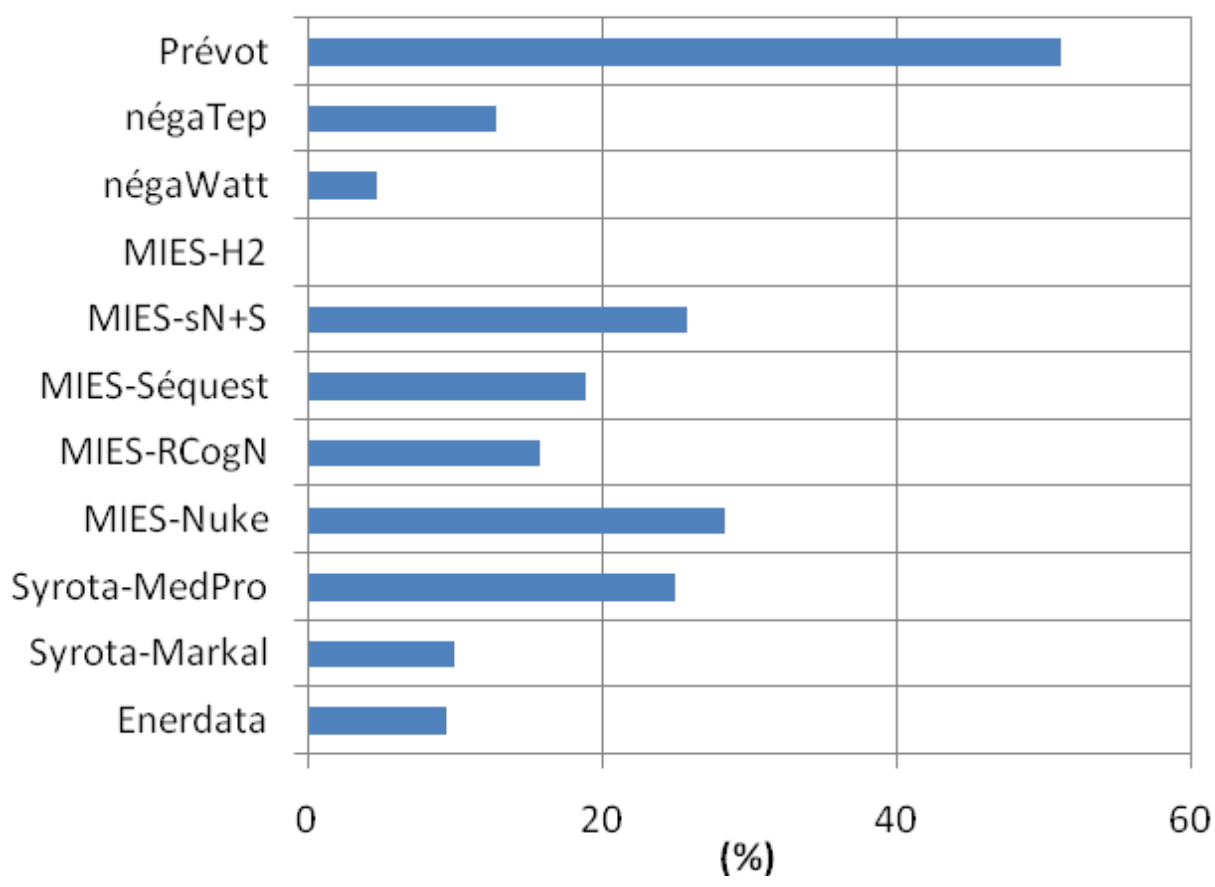

\section{Le secteur des bâtiments}

Dans le secteur des bâtiments ${ }^{10}$, la réduction du contenu carbone de l'énergie finale joue un rôle prépondérant par rapport à la baisse de la consommation d'énergie finale. Ceci est dû, dans la plupart des cas, à la pénétration de l'électricité dans le secteur avec le déploiement du chauffage électrique adossé à de l'électricité nucléaire (Figure 3). Le scénario qui va le plus loin dans cette option est le scénario Syrota-Markal avec $70 \%$ environ de l'énergie finale du secteur consommée sous forme d'électricité (31\% aujourd'hui). Le scénario négaWatt se distingue par la faible part de l'électricité d'origine non renouvelable dans le secteur résidentiel/tertiaire. La seule électricité d'origine non renouvelable y est de l'électricité cogénérée et provenant de cycles combinés au gaz à haut rendement.

Alors que tous les scénarios insistent sur la nécessité d'un programme ambitieux de rénovation thermique de l'habitat, les diminutions de la consommation d'énergie finale par habitant sont très variées. Les deux scénarios qui vont le plus loin dans cette décroissance sont ceux qui vont également le plus loin en termes de réduction des émissions du secteur. Enerdata et négaWatt représentent un programme ambitieux de constructions neuves et de rénovation thermique de l'habitat, mais aussi une large diffusion des réseaux de chaleur et des chauffe-eau solaires. À l'autre extrême, le scénario MIES-sN+S ne permet pas d'aller plus loin qu'une stabilisation des émissions.

D'autre part, les deux scénarios supposant une sortie du nucléaire montrent des réductions de $\mathrm{CO}_{2}$ très variés : dans négaWatt les réductions atteignent $82 \%$ et dans le MIES-sN+S seulement 10\%. Dans le scénario négaWatt, ce sont les énergies renouvelables 
qui remplacent les énergies fossiles et l'électricité nucléaire, alors que dans MIES-sN+S c'est la technologie CCS.

Figure 3 : part de l'électricité d'origine non renouvelable dans l'énergie finale consommée dans le secteur résidentiel/tertiaire

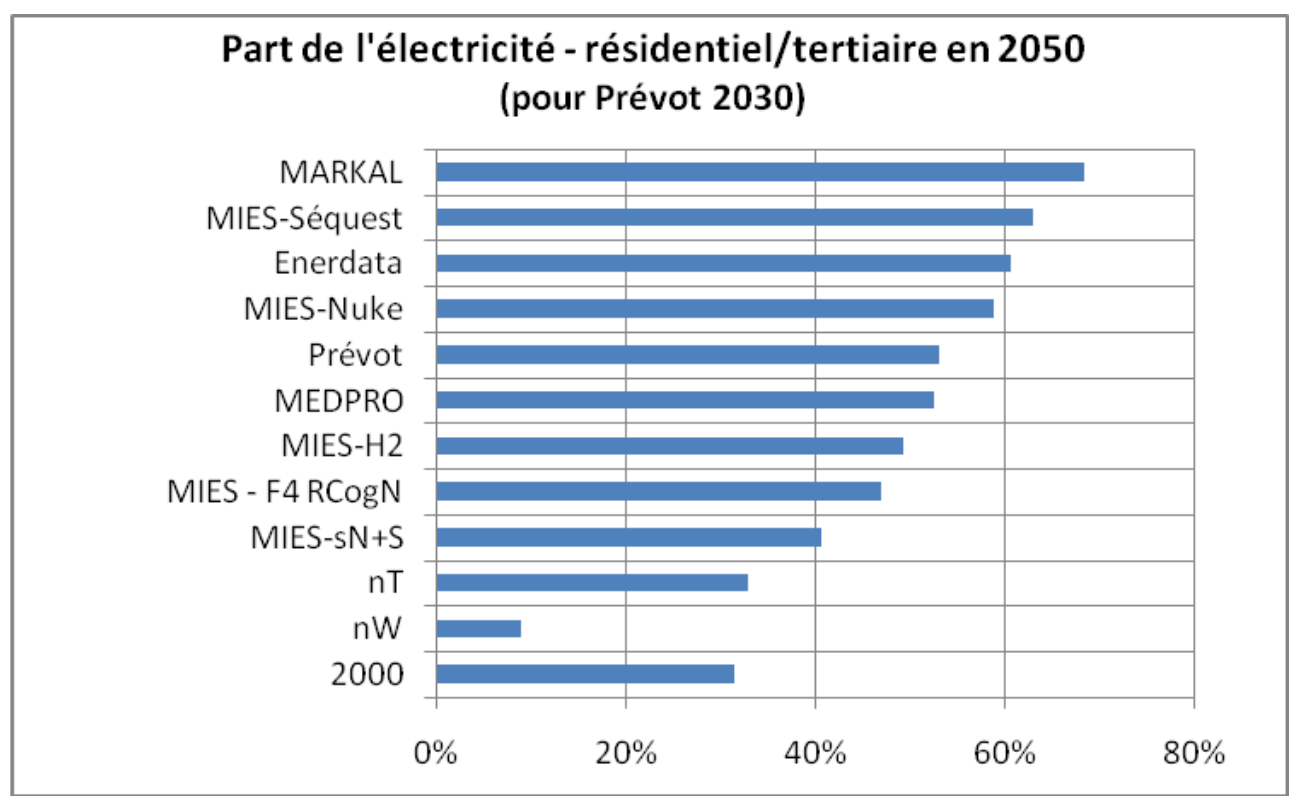

\section{Le secteur de l'industrie}

C'est dans ce secteur que les résultats sont les plus contrastés : le scénario MIES-sN+S prévoit une augmentation des émissions du secteur de $30 \%$, tandis que dans le scénario négaWatt les émissions du secteur diminuent de $77 \%$. Que ce soit en termes d'évolution du contenu carbone de l'énergie finale ou d'évolution de la consommation d'énergie finale par habitant, les résultats varient beaucoup. Deux visions sur l'évolution du secteur industriel semblent donc s'opposer: d'un côté MIES-sN+S, MIES-Séquest ${ }^{11}$, MIES-nuke, MIES-H2, MIES-RCogN et Syrota-Markal qui projettent une augmentation de l'énergie finale consommée par habitant et de l'autre côté Enerdata, négaTep, Med-Pro et négaW.

Cette forte dispersion des résultat peut résulter de plusieurs facteurs de nature différente. Tout d'abord, la part de la production domestique dans la consommation de biens des ménages et des entreprises. Comme déjà mentionné précédemment, une contrainte carbone en France peut conduire à reporter une partie de la production industrielle dans des pays à moindre contrainte, ce qui conduirait à diminuer l'activité industrielle et donc les émissions attenantes. Cette dimension ne peut donc se postuler de manière déconnectée d'une réflexion sur l'économie mondiale et sur l'évolution du coût des facteurs de production (et pas uniquement le facteur énergie) dans chacune région du monde. Ensuite, il faut tenir compte de l'évolution structurelle de l'industrie française vers des marchés spécifiques porteurs en termes d'innovation qui peuvent être, suivant le contexte mondial, plus ou moins émettrices de GES. Enfin, il y a l'influence des modes de développement et les styles de vie selon que les préférences des consommateurs s'orientent vers plus de sobriété ou continuent sur des niveaux de consommation matérielle croissants. Ceci renvoie aux valeurs d'une société Facteur 4: une société Facteur 4 est-elle compatible avec un mode de vie consumériste? 
39 De ce fait, représenter l'évolution des émissions de $\mathrm{CO}_{2}$ dans le secteur industriel nécessite une compréhension fine des dynamiques industrielles et économiques au niveau français, européen et mondial. Ceci ne pourrait se faire qu'avec un fort niveau de désagrégation nécessitant des données et potentiels techniques des différentes branches industrielles alors que dans la majorité des scénarios, la représentation de l'industrie reste à un niveau très fruste. Néanmoins, le devenir du secteur industriel est central car il interroge le contenu même de la croissance économique ainsi que la notion de biens essentiels propre à l'approche de négaWatt.

\subsection{Quel paysage énergétique?}

Les figures 4 et 5 montrent la diversité des mutations envisagées pour la projection de l'énergie primaire en 2050.

Figure 4 : Répartition du mix énergétique dans l'énergie primaire en volume

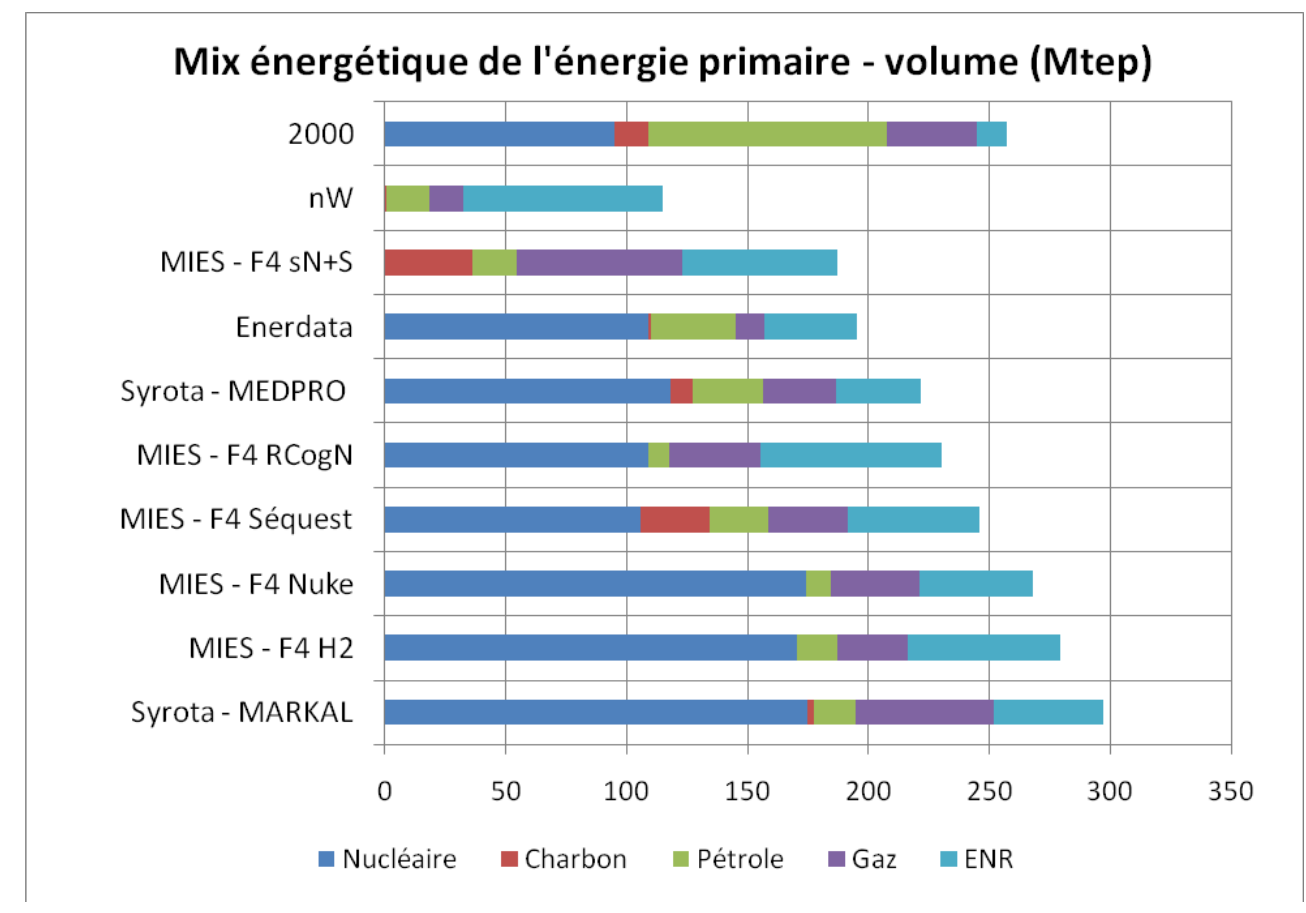


Figure 5: Répartition du mix énergétique dans l'énergie primaire en pourcentage

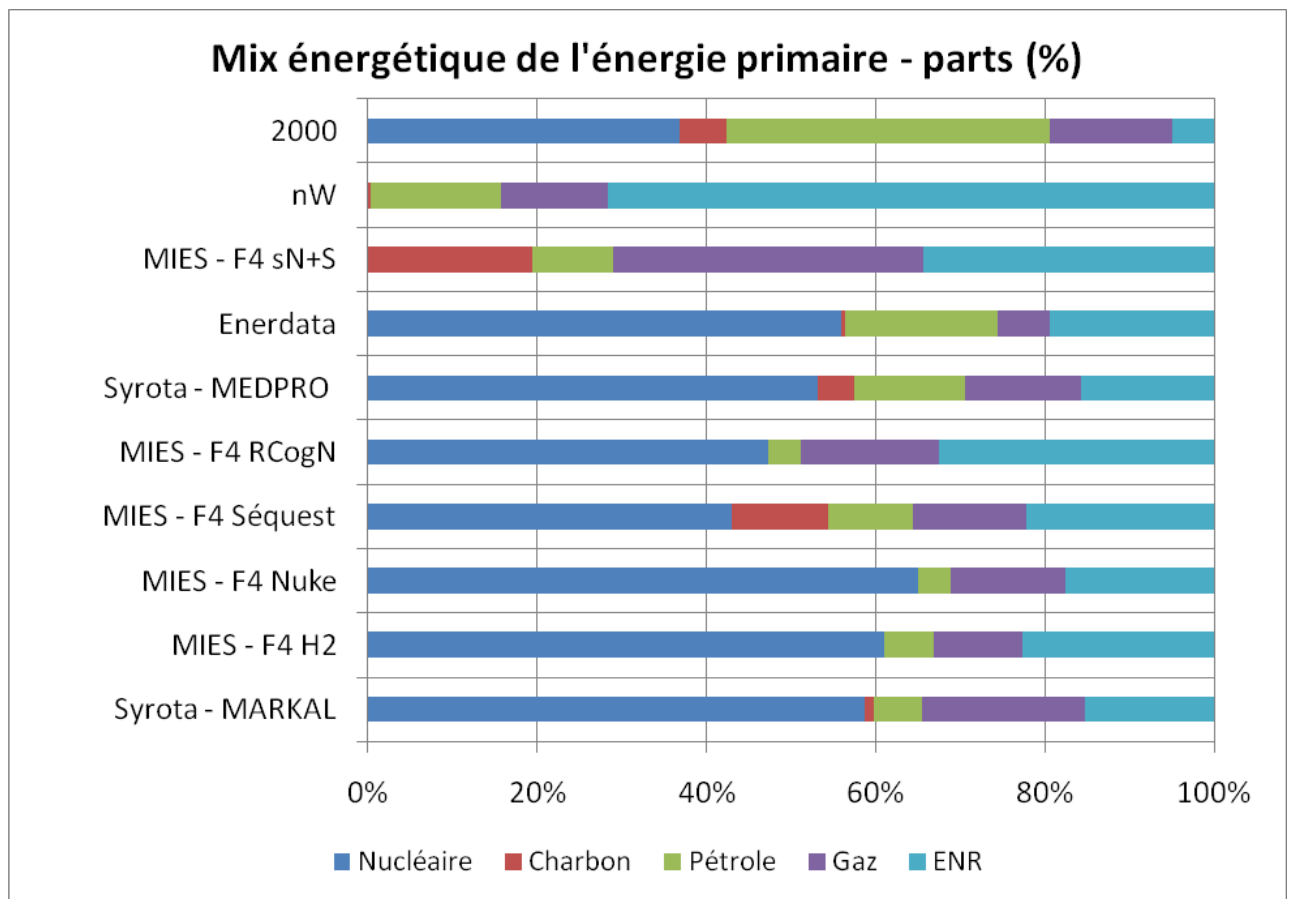

41 Tout d'abord, un des points saillants de l'analyse est la tendance à une substitution vers l'électricité de l'énergie consommée pour les transports (véhicules électriques) voire pour le chauffage et l'industrie. Ceci combiné à la vision spécifique à chacun des scénarios quant à la place laissée au nucléaire semble déterminer le niveau d'énergie primaire. Ainsi, seuls les trois scénarios avec un redéploiement massif du nucléaire (MIES F4-H2, MIES Nuke, Syrota-Markal) conduisent à une croissance de l'énergie primaire. Le maintien du nucléaire à un niveau comparable au niveau actuel induit une légère baisse de la production d'énergie primaire, tandis que les deux scénarios avec sortie du nucléaire (négaWatt et MIES $\mathrm{sN}+\mathrm{S}$ ) correspondent aux deux scénarios avec la plus forte baisse d'énergie primaire consommée.

Figure 6 : Mix technologique de la production d'électricité en 2050 dans les différents exercices, en parts de marché à gauche et en valeur absolue (Twh) à droite
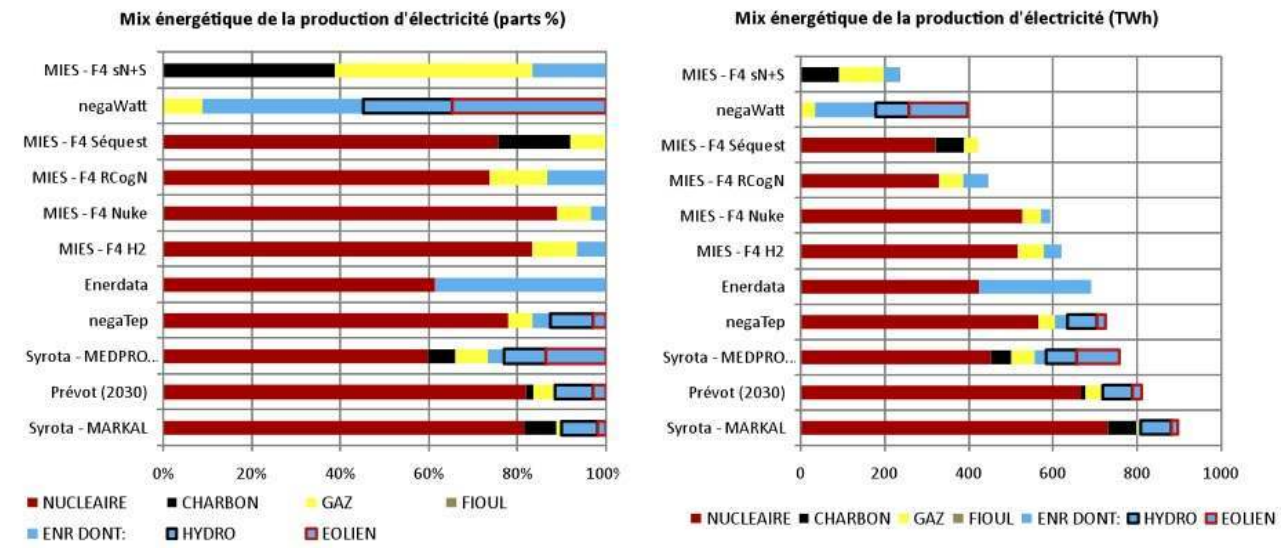

envisage la coexistence du nucléaire et des ENR à grande échelle (40\% du mix). Ceci interroge néanmoins sur le niveau de complémentarité qui peut exister entre d'un côté, une technologie peu flexible en terme de niveau de production et de l'autre des technologies qui peuvent si elles sont intermittentes (éolien et solaire) avoir besoin de s'appuyer sur d'autres sources de production d'électricité flexibles (en général on considère que les centrales cycles combinées au gaz sont bien plus flexibles que le nucléaire et que de ce fait ils seraient davantage complémentaires d'un déploiement éolien).

Au-delà, les scénarios avec prédominance du nucléaire posent la question de la modélisation des usages saisonniers de l'électricité (notamment chauffage) et de l'optimisation du mix de production qui en résulte. Par exemple, certains scénarios n'incluent pas le recours saisonnier aux moyens centralisés de production thermique à flamme ou le potentiel de lissage de la demande d'électricité. Dans de nombreux exercices, la représentation des sources d'énergie décentralisée n'est pas assez précise, en particulier pour celles présentant un très bon rendement énergétique. Ceci conduit à un report massif des besoins énergétiques vers des solutions centralisées en particulier vers le nucléaire, sélectionné pour son coût de production considéré comme faible. Il est donc nécessaire de considérer davantage les deux variables importantes que sont le niveau de la capacité d'ajustement du système et la diversité des sources à mettre en œuvre. Enfin, la réflexion sur l'évolution du système électrique français ne peut plus être isolée des 
problématiques d'interconnexions, de smart grid, d'exportations et d'importations de l'électricité sur la plateforme européenne dans son ensemble.

La nécessité d'avoir recours à des technologies non ou faiblement carbonées explique, outre la place du nucléaire dans le mix énergétique :

1. La forte diminution de la consommation de pétrole dont l'utilisation sera au moins 3 et jusqu'à 20 fois inférieure au niveau actuel ;

2. L'interdiction du charbon sauf à recourir au CCS (MIES SN+S ou MIES RCogN);

3. La place du gaz, modérément émetteur de $\mathrm{CO}_{2}$ comparé au pétrole ou au charbon, qui se déploie grâce aux bons rendements de la cogénération ;

4. La part des ENR dans le mix énergétique qui varie beaucoup mais avec une contribution minimum significative de 50 Mtep. En dehors des ENR électriques, si le scénario ne projette pas de déploiement des véhicules électriques (ou à hydrogène), il est incontournable (en dehors de la diminution de la demande de transports) de développer des agrocarburants de deuxième génération qui devront présenter les meilleurs bilans énergétiques et $\mathrm{CO}_{2}$ possibles.

\section{Quel rôle pour la scénarisation?}

La description des approches et des résultats des différents exercices de réduction d'émissions de $\mathrm{CO}_{2}$, plutôt que de fournir une vision claire du Facteur 4, élargit le spectre des questions. Un seul scénario aboutit réellement à un facteur 4 , le scénario négaWatt avec sortie du nucléaire, alors que les scénarios avec maintien ou renforcement de la place du nucléaire butent sur un facteur 3. Que doit-on conclure de ces résultats? Dans cette dernière partie nous nous interrogeons sur la nature des limites des scénarios et des modèles utilisés et sur le rôle de ces exercices.

\subsection{Les limites des exercices analysés}

Qu'y a-t-il dans la boîte noire des modèles d'ingénieur ?

Tout d'abord, la complexité des modèles, la diversité de leurs hypothèses et la dispersion de leurs résultats sont susceptibles de rendre inaudibles ou suspects les enseignements potentiels des travaux numériques. Certes, le but de tels exercices n'est pas de prévoir le scénario le plus probable, mais bien de montrer, selon des hypothèses technologiques, des mix énergétiques cohérents avec une contrainte carbone. Pour le béotien, les différences dans les résultats sont difficilement explicables d'autant plus si le seul message communiqué se résume à la description d'un mix technologique possible et d'un niveau de réductions d'émissions en 2050 sans que les hypothèses sous-jacentes ne soient réellement palpables. Quels sont les déterminants des résultats obtenus? Quelles sont les contributions respectives aux réductions d'émissions, d'un progrès technique autonome qui ne ferait que suivre une tendance passée, d'hypothèses sur l'évolution du prix du pétrole, d'un progrès technologique induit par des politiques climatiques ou de changements de comportement du fait de politiques climatiques ou de l'évolution des préférences des consommateurs?

51 Or, les différences sur les perspectives de pénétration des différentes technologies relèvent de justifications de natures bien différentes : purement technologique, politique voire idéologique ou encore méthodologique. La constitution du mix énergétique relève 
d'une expertise difficilement séparable des opinions du modélisateur ou du commanditaire ; tel fera un scénario 'doux' mobilisant les énergies ENR parce qu'il considèrera que le nucléaire ne répond pas aux critères de durabilité, tel autre mettra en scène la nouvelle corne d'abondance que constituerait une société de l'hydrogène ou du tout électrique adossé au nucléaire civil. Des différences apparaissent également sur les perspectives d'amélioration des technologies et de gains en efficacité énergétique en fonction de dires d'experts plus ou moins volontaristes ou plus ou moins récents. Ainsi le scénario négaWatt prévoit que la voiture à moteur à explosion consommera $3,3 \mathrm{~L} / 100 \mathrm{~km}$ en 2050 quand le rapport Syrota table sur un niveau moyen du parc de 5,9L/100km. Qui a raison ? À quoi sont dues ces différences d'appréciations? Quel est l'impact de ce type de différences sur les résultats? D'autres différences proviennent de la part du gisement total d'efficacité énergétique qui sera effectivement mis en œuvre non pas d'un point de vue technique, mais du point de vue de l'ambition et de l'efficacité des mesures appliquées. Il en est ainsi des potentiels d'économie d'énergie pouvant être mis en œuvre dans le cas d'un programme de rénovation thermique de l'habitat. Tous les scénarios s'accordent sur la nécessité d'un tel programme, mais aucun ne met en œuvre ce programme de la même manière. Au final, les performances chiffrées sont fort disparates: nombre de rénovations par an, niveau de consommation visé lors de la rénovation. Un des rôles de ces exercices devrait justement être d'évaluer selon le type de mesure par secteur (réglementation vs. mesure fiscale), les réductions d'émissions et les coûts associés. Enfin, dans de nombreux cas, la sobriété énergétique qui permet de diminuer les gaspillages n'est pas mentionnée, car agrégée aux gains dus à l'efficacité énergétique. Le scénario négaWatt est le seul à la considérer en tant que telle, de manière indépendante, alors que ce sont donc des gains énergétiques gratuits, atteignables immédiatement à la différence des mesures d'efficacité énergétique qui ne pénètrent qu'au rythme du renouvellement des parcs techniques. Toutefois, la sobriété énergétique suppose une évolution des comportements. Nous sommes aujourd'hui incapables de détailler les mesures nécessaires à un tel changement.

Dans ces conditions, il n'est pas difficile de comprendre que les résultats de ces exercices d'ingénieur apparaissent comme issus de véritables boîtes noires, difficilement appropriables en dehors d'une communauté de gens avertis (modélisateurs, institutions et commanditaires). Ces travaux ne font que révéler la vision d'un cercle restreint de personnes. Leur rôle et leur légitimité en sont donc limités.

\section{Un système énergétique peut-il être indépendant du système économique ?}

53 Les scénarios relatés ici reposent sur des modèles dits bottom-up ou sur des analyses de données technologiques. Riches en informations technologiques, ils utilisent un langage proche de celui de l'ingénieur (expression en quantités physiques des besoins de confort thermique, d'éclairage, de mobilité ou de force motrice). Leur composante économique se réduit à la projection d'un scénario de croissance économique simple (le PIB, sa répartition sectorielle et le revenu des ménages) et à la formulation d'hypothèses sur les coûts des techniques et des énergies fossiles. Ceci pose plusieurs problèmes. 1) Penser ces systèmes techniques indépendamment de leur contexte économique et social manque de réalisme. Certaines projections peuvent par exemple ne correspondre à aucun état possible de l'économie du fait de contraintes sur les investissements.

2) Représenter les trajectoires de croissance sous forme de tendance régulière plus ou moins pentue est loin d'être anodin pour l'évaluation des coûts des politiques climatiques 
et pour la conduite du débat public. Les modélisateurs du long terme ont l'habitude de fonder leurs simulations sur des hypothèses régulières sans heurt, en argumentant que les fluctuations de court terme n'affectent pas les tendances de long terme ; négligeant les possibles instabilités observées dans la réalité concernant les marchés énergétiques, les déséquilibres extérieurs chroniques ou encore le poids des dettes nationales. Or, les chocs possibles sur les marchés de l'énergie, par une flambée directe des prix ou par une insuffisance de l'approvisionnement, peuvent avoir des impacts macroéconomiques dépassant largement la sphère énergétique. Les exemples les plus évidents sont les chocs pétroliers de 1973, 1979, et les phases de croissance rapide des prix comme entre 2004 et 2008. Il est crucial de pouvoir représenter ce type de chocs et les réactions d'ajustement pouvant entraîner des bifurcations technologiques (le choix du nucléaire en France à la suite des chocs pétroliers), parce que les politiques énergétiques ou climatiques visent précisément à minimiser les risques liés aux deux premiers types de chocs : recherche d'une plus grande indépendance ou frugalité énergétique, réduction des émissions de GES pour limiter le changement climatique, mesures d'adaptation préventive face aux événements climatiques. Du point de vue de l'analyse coûts-bénéfices des politiques éventuelles, les bénéfices des chocs évités, ou dont la propagation dans l'économie est atténuée grâce à ces politiques, peuvent représenter une part significative des gains totaux. Les négliger conduit par exemple à conclure que le changement climatique ne menace que quelques pourcents de PIB à un horizon de plusieurs décennies alors que de nombreux chocs successifs peuvent entraîner une amplification des pertes de manière significative (Ambrosi, 2004). Or, il n'est pas inutile de rappeler qu'aucun des exercices passés en revue ne prend en compte les impacts du changement climatique à la fois sur l'évolution des préférences des consommateurs (loisirs, localisation...), des productivités sectorielles (sur l'agriculture ou même sur les pertes de performances des centrales nucléaires si les canicules sont amenées à se multiplier), des impacts d'événements extrêmes, de la mise en œuvre de mesures d'adaptation aux côté des mesures de réduction des émissions. La difficulté de prévoir et quantifier ces impacts conduit à cette simplification. Même si l'on peut penser que les impacts seront sans doute limités en France métropolitaine, il n'est pas inutile de renvoyer le lecteur vers l'évaluation des dommages menée par l'ONERC (2009).

55 3) Il est irréaliste de penser que les stratégies Facteur 4 pourraient se limiter à l'introduction d'un prix du carbone qui rendrait possible à lui seul à la fois la pénétration des technologies non carbonées et les changements de comportement des consommateurs sur la totalité de leur consommation. Le champ d'action est bien plus diversifié : règlementation, politiques d'infrastructures et aménagement du territoire, politiques sectorielles, subvention aux ENR (tarifs d'achat). Ainsi, Combet et al. (2009a) montrent, pour les transports, que des politiques dédiées ${ }^{14}$ permettent de diviser par deux le niveau nécessaire de la taxe pour atteindre le Facteur 4 en 2050. Ils utilisent pour cela un modèle (dit hybride) représentant l'évolution des systèmes technologiques en cohérence avec les contraintes macroéconomiques (modèle Imaclim-R).

4) Les politiques climatiques ne peuvent être déconnectées des autres politiques menées dans d'autres domaines de l'intervention publique. Ainsi, la taxe carbone offre l'opportunité d'un effet de levier sur la fiscalité. D'influents travaux antérieurs sur la notion de double dividende (Bovenberg et Mooij, 1994) ont mis en évidence qu'utiliser les revenus de la taxe carbone pour baisser la fiscalité sur le travail permettait de réduire les effets économiques négatifs de cette taxe carbone (Combet et al., 2009b). Il est donc 
nécessaire de disposer d'outils montrant les interactions entre systèmes techniques et contraintes économiques afin d'évaluer les stratégies d'atteinte du Facteur 4.

\subsection{La scénarisation comme outil de construction du consensus et d'appropriation sociale}

Nous l'avons vu, les modèles ou les scénarios tels qu'ils sont utilisés aujourd'hui apparaissent comme des boîtes noires pour qui ne fait pas partie du cercle proche du modélisateur. Ceci pose un problème d'autant plus important qu'il n'existe encore que peu de scénarios réalisés indépendamment de l'Etat et à un niveau politique suffisant pour porter le débat public. Ainsi, au travers des 7 exercices présentés ici, 4 sont issus de commandes institutionnelles (la MIES, la DGEMP, la Commission Syrota) avec un fort argument d'autorité. Or, d'une part, les messages portés par ces exercices ne sont pas anodins - une approche purement technologique, l'impossibilité à atteindre le Facteur 4, un fort déploiement du nucléaire et finalement un rôle de l'État en retrait par rapport à l'action du marché et des acteurs privés... - et, d'autre part, s'ils doivent servir à la construction de la décision publique, ils n'ont que la légitimité d'une communauté restreinte. Le risque est alors d'imposer des politiques climatiques par le haut et de manière déconnectée des autres enjeux de politique française alors que le climat est un problème de société à plusieurs titres. Il est impossible de parler de politiques climatiques sans parler de politique en matière d'emploi (la rénovation thermique de l'habitat peut créer jusqu'à 250.000 emplois, $c f$. Demailly et Quirion, 2008), de politique sociale avec l'apparition de la "précarité énergétique » ou de politique en matière de formation, d'infrastructures ou encore de logements.

Les outils de modélisation pourraient donc servir de boîtes de dialogue et d'outils de confrontation entre les parties prenantes issues de la société civile, des acteurs économiques et du monde politique de manière à élaborer conjointement une trajectoire cohérente et acceptable par les plus nombreux d'entre nous, définir les réallocations sectorielles, les compensations nécessaires.

L'enjeu serait alors d'accompagner et d'alimenter les travaux de modélisation par des échanges au sein d'une plateforme entre modélisateurs, économistes, technologues, sociologues et représentants de la société civile. Ceci nécessite de casser les barrières existant entre les champs disciplinaires. L'enjeu est aussi que que les modèles, au-delà d'une seule projection technologique, représentent de manière cohérente et conjointe l'évolution des systèmes techniques et les contraintes économiques. Il convient aussi d'ouvrir les boîtes noires que sont les modèles et d'établir, sous la forme d'interface utilisateurs, des plateformes d'échanges sur les données et hypothèses des modèles et des scénarios de manière à ce que chacun puisse éventuellement faire lui-même son propre scénario Facteur 4 et s'approprie les barrières, les incontournables, les impacts de tel ou tel politique climatique. Il faut enfin rendre parlantes les données, les variables de scénarios et les sorties des modèles. Un dialogue élargi ne sera possible que si les données du modèle peuvent être traduites à la fois en données économiques (prix, niveaux de taxe, taux d'emploi, parts de budgets des ménages...) et en données physiques énergétiques (Mtep, TWh...) de manière à représenter l'évolution des modes de vies qu'ils soient choisis ou contraints: la forme des villes, le temps de transport quotidien, la distance domicile-travail, le nombre de $\mathrm{m}^{2}$ de logement par ménage, les taux d'équipement... 
60 Ce n'est qu'à ce titre qu'une négociation objective pourra avoir lieu entre les gagnants d'un scénario et les perdants. Un tel exercice aurait été fort utile pour faire émerger une taxe carbone efficace (niveau de prélèvement) et juste (redistribution, exonérations).

\section{Conclusion}

61 Les différents scénarios et exercices de modélisation sur le Facteur 4 existants en France s'appuient tous sur des modèles représentant les systèmes techniques de manière largement indépendante non seulement $\mathrm{du}$ système économique, mais aussi de l'évolution des préférences des consommateurs dans un monde sous contrainte environnementale. De ce fait, les exercices que nous avons analysés ici peuvent difficilement proposer des stratégies permettant les bifurcations nécessaires pour répondre aux impératifs du Facteur 4. Nous proposons donc un cahier des charges pour une modélisation devant permettre à la fois de représenter les interactions entres économie, systèmes techniques et modes de vie, mais aussi devant servir de plateforme aux échanges entre sociologues, modélisateurs, économistes, technologues et société civile. Ce n'est qu'à ce titre que de tels exercices acquerront une légitimité renforcée : en devenant des outils d'aide à l'appropriation sociale et politique.

\section{BIBLIOGRAPHIE}

Acket C., Bacher P.(Association Sauvons le Climat), 2007, Diviser par 4 nos rejets : le scénario Negatep

Allen P., Helweg-LarsenT., Bull J., 2007, « ZerocarbonBritain », CAT Center for alternative technologies.

Ambrosi P., 2004, Amplitude et calendrier des politiques de réduction des émissions face aux risques climatiques : leçons des modèles intégrés, Thèse de doctorat de science économique, Hourcade, J-C (dir.), Paris, EHESS.

Association négaWatt, 2006, Scénario négaWatt 2006, pour un avenir énergétique sobre, efficace et renouvelable, document de synthèse, Paris, 16 décembre 2005.

de Boissieu C. (sous sa présidence), 2006, « Division par quatre des émissions de gaz à effet de serre de la France à l'horizon 2050 », Groupe Facteur 4, http://

www.ladocumentationfrancaise.fr/rapports-publics/064000757/index.shtml

Bovenberg A. L, de Mooij R. A., 1994, «Environmental Levies and Distortionary Taxation », American Economic Review, 84 (4), p. 1085-1089.

Chambolle T., 2007, Partie 5 - Scénarios énergétiques, Rapport du Groupe de Travail 5, Président Thierry Chambolle, rapporteur Hervé Pouliquen, in Perspectives énergétiques de la France à l'horizon 2020-2050, rapport d'orientation, Centre d'Analyse Stratégique. http:// www.ladocumentationfrancaise.fr/rapports-publics/074000660/index.shtml

Combet E., Ghersi F., Guivarch C., 2009a, Les transports et le Facteur 4 - Entre diversification des signaux et réforme fiscale, Rapport final PREDIT GO n¹1 Politique des transports, 26 juin, 61 pp. 
Combet E., Ghersi F., Hourcade JC., 2009b, « Taxe carbone, une mesure socialement régressive? Vrais problèmes et faux débats », WP CIRED.

Commission Energie, 2007, Les perspectives énergétiques de la France à l'horizon 2020-2050

Demailly D., Quirion P., 2008, «-30\% de $\mathrm{CO}_{2}=+684000$ emplois pour la France », CIRED-WWF, http://www.centre-cired.fr/perso/quirion/quirion_emploi_wwf.pdf

Dessus B., 2008, « Effet de serre, n'oublions pas le méthane », La Recherche, numéro 417

Enerdata-Lepii, 2005, Étude pour une prospective énergétique concernant la France, Observatoire de l'Energie, Direction Générale de l'Energie et des Matières Premières

Hourcade, J. C., 2007, «Les modèles dans les débats de politique climatique : entre le Capitole et la Roche tarpéienne? », in A. Dahan-Dalmédico (éd.), Les modèles du futur, La Découverte, Paris.

Nakano S., Okamura A., Sakurai N., Suzuki M., Tojo Y., Yamano N.,2009, « The Measurement of CO ${ }_{2}$ Embodiments in International Trade: Evidence from the Harmonised Input-Output and Bilateral Trade Database », OECD Science, Technology and Industry Working Papers, 2009/3, OECD publishing, ( OECD

Nitsch, J., 2008, Leitstudie 2008, - Further development of the 'Strategy to increase the use of renewable energies' within the context of the current climate protection goals of Germany and Europe, Study commissioned by the German Federal Ministry for the Environment, Nature Conservation and Nuclear Safety (BMU), octobre, http://www.bmu.de/english/ renewable_energy/downloads/doc/42726.php

ONERC (Observatoire National des Effets du Réchauffement Climatique), 2009, «Évaluation du coût des impacts du changement climatique et de l'adaptation en France », septembre

Prévot H., 2004, « Politique énergétique nationale et lutte contre l'effet de serre », La Revue de l'énergie, n554, p. 69-78.

Prévot H., 2007, Trop de pétrole - énergie fossile et réchauffement climatique, Le Seuil, Paris

Quinet, E. (sous la présidence de), 2009, La valeur tutélaire du carbone, Centre d'analyse stratégique, $\mathrm{n}^{\circ} 16$, [en ligne] :

http://www.strategie.gouv.fr/IMG/pdf/Rapp_16_VTC_web.pdf

Radanne P., 2004, « La division par 4 des émissions de dioxyde de carbone en France d'ici 2050 », Mission Interministérielle de l'Effet de Serre, 36 Syrota, J, 2007, « Perspectives énergétiques de la France à l'horizon 2020-2050 », Centre d'analyse stratégique, http://www.strategie.gouv.fr/ article.php3?id_article $=675$

Virage énergie, 2008, « Énergie de demain », Association Virage Énergie, http://www.virageenergie-npdc.org

\section{NOTES}

1. La loi POPE inscrit bien l'objectif de $3 \%$ de diminution annuelle des GES. Toutefois, à part une unique citation concernant les aérosols, dans l'article 2 de la loi, il n'est fait mention des différents GES qu'à des fins énergétiques. Cf. Dessus 2008 qui développe l'exemple du méthane, un autre GES.

2. Modéliser l'évolution des émissions de $\mathrm{CO}_{2}$ issues de la combustion des énergies fossiles nécessite de comprendre et de représenter les déterminants de l'évolution du mix énergétique. Modéliser l'évolution des autres GES et notamment des GES issus de l'agriculture et des usages 
des sols relève de déterminants et de dynamiques très différentes, ce qui explique le fait que les exercices présentés ici ne représentent qu'une partie des émissions.

3. Cf. Chambolle 2007, partie 5 du rapport sur les scénarios énergétiques.

4. Ce scénario régional est construit sur la base d'hypothèses Enerdata régionalisées.

5. Deux grandes familles de modèles ont émergé dans le champ de la prospective énergétique : d'un côté des modèles d'ingénieurs dits « bottom-up », de l'autre, les modèles dits « top down ». Les modèles bottom-up reposent sur une représentation précise du système de production et de transformation de l'énergie, avec des hypothèses exogènes sur la disponibilité des ressources naturelles, la croissance et la demande finale de services énergétiques. Les modèles top-down sont des modèles captant les mécanismes économiques, les rétroactions macroéconomiques : réaction de la demande au prix de l'énergie par exemple. Les modèles «top-down » sont critiqués pour leur représentation peu tangible et peu maîtrisée des possibilités techniques, par leur caractère agrégé et purement monétaire, tandis que les modèles «bottom-up » ignorent le rôle du système économique entier dans l'évolution des signaux-prix et des contraintes de budget qui sont déterminantes dans la bifurcation des systèmes techniques. Cf. Hourcade 2007 sur les modèles dans les débats de politiques climatiques.

6. Il faut distinguer énergie finale et énergie primaire. L'énergie primaire est l'énergie brute telle qu'elle est disponible dans la nature avant toute transformation. L'énergie finale est l'énergie que l'on consomme c'est à-dire l'énergie primaire que l'on a transformée dans les centrales de production d'électricité, dans les raffineries... Le rapport entre les deux définit le rendement du système énergétique.

7. Le premier scénario Prévot a été réalisé en 2004. À cette époque le prix du baril était aux alentour de $25 \$ / \mathrm{bl}$.

8. La technologie Coal To Liquid (CTL) est une technologie backstop, dans le jargon des modélisateurs, c'est-à-dire que le coût de production de carburant avec du CTL borne le prix du carburant. A partir du moment où le prix du baril de pétrole atteint le coût de production du CTL (qui ne pourra que décroitre puisque les ressources en charbon sont abondantes), il y aura une substitution du pétrole vers cette technologie.

9. La date de maturité de la technologie CCS fait l'objet d'incertitudes très fortes, malgré les investissements importants réalisés dès à présent dans des projets pilotes. La Commission Européenne affiche sa volonté de soutenir la mise en place de 12 démonstrateurs industriels de CCS d'ici 2015. La France est présente dans cette course industrielle, avec le lancement par l'ADEME en 2009 d'une série de projets de démonstrateurs de recherche. En France, les projets pilotes sont les projets Total à Lacq (Pyrénées Atlantiques), Veolia Environnement à Claye Souilly (Seine et Marne), Arcelor Mittal à Florange (Moselle). Au Havre un projetvise à étudier la mise en place d'un réseau mutualisé de transport des fumées.

10. Dans le secteur des bâtiments, les deux dimensions autour desquelles s'organisent les consommations d'énergie et les émissions de $\mathrm{CO}_{2}$ induites relèvent de dynamiques indépendantes : le chauffage des bâtiments et l'électricité spécifique qui connaît une très forte croissance. Réduire les émissions de $\mathrm{CO}_{2}$ pour le chauffage des bâtiments nécessite à la fois des programme ambitieux de rénovation thermique de l'habitat et de développement de formes d'énergie non carbonées. Réduire les émissions induites par la hausse des consommations d'électricité spécifique nécessitera de généraliser les étiquettes énergie et de renforcer la réglementation sur les appareils consommateurs d'électricité. Dans les scénarios étudiés ici, l'accent a surtout été mis sur le premier volet de ces consommations et il est difficile de distinguer à leur lecture les réductions de consommation et d'émissions de manière séparée. L'étude des déterminants de la croissance de l'électricité spécifique est délicate du fait de l'émergence continue de nouveaux usages.

11. Les scénarios avec séquestration géologique (MIES-sN+S, MIES-Séquest) du carbone permettent de relâcher la contrainte carbone dans le secteur industriel. 
12. En prenant comme hypothèse un temps de fonctionnement moyen des éoliennes de 2200 heures par an, une production de 170 TWh correspond à une puissance installée de $62 \mathrm{GW}$. Pour donner un ordre de grandeur, la puissance installée en 2010 est de 4,6 GW avec 2600 éoliennes sur le territoire français et l'objectif retenu par le Comité Opérationnel sur les énergies renouvelables du Grenelle de l'Environnement est de $25 \mathrm{GW}$ en 2020 qui devraient être fournis par 8000 éoliennes (dont 850 en mer). La valeur atteinte dans le scénario négaWatt ne parait donc pas inatteignable. En revanche, ceci présuppose la levée des barrières administratives adoptées dans le cadre du Grenelle de l'Environnement sur le déploiement de l'éolien, tout particulièrement la soumission des projets éoliens à la procédure ICPE des installations classées.

13. Malheureusement, il est difficile d'aller bien au-delà dans l'analyse comparative du déploiement des ENR car les différentes technologies ENR ne sont souvent pas détaillées.

14. Principalement une politique d'infrastructures doublée d'une politique de réorganisation des chaînes logistiques de production et de distribution visant à réduire le 'contenu transport' des consommations finales et une réorientation de l'urbanisme vers une limitation des besoins de mobilité.

\section{RÉSUMÉS}

Plusieurs exercices de prospective de long terme ont été élaborés pour définir les trajectoires énergétiques permettant de respecter le Facteur 4 en France. Sept ont été identifiés et sont analysés à la fois du point de vue méthodologique et des résultats. Une réflexion est alors menée sur le rôle de la scénarisation. Paradoxalement, les résultats de ces analyses montrent qu'un seul des scénarios permet d'atteindre le Facteur 4, démontrant ainsi les limites de ces études. D'un point de vue méthodologique, les modèles d'ingénieur utilisés apparaissent comme des boites noires, avec leurs propres hypothèses technologiques et qui rendent les résultats peu appropriables par le non-spécialiste. L'enjeu est donc d'accompagner les travaux de modélisation par des échanges au sein d'une plateforme entre modélisateurs, économistes, technologues, sociologues et représentants de la société civile. Pour renforcer leur légitimité, ces scénarios doivent ainsi participer à l'appropriation sociale et politique des résultats scientifiques.

Seven long-term prospective studies representing the energy trajectories consistent with a Factor Four, i.e. a 75\% reduction of greenhouse gases emissions in 2050 in France have been identified. We analyze their methodology and the high dispersion of results. Then we discuss the role of scenario-making. Among them, only one of the scenarios achieves the Factor Four, thereby showing the limitations of these studies. On the methodological side, the engineering models used appear as black boxes, each using their own technological hypotheses and not readily understandable by the non-specialist. Therefore, exchanges between modelers, economists, technologists, sociologists and representatives of the civil society are a key factor for these scenario elaboration as their legitimacy stems from social and politic appropriation of scientific results. 


\section{INDEX}

Keywords : carbon price, climatic policies, emissions, energy mix, energy scenarios, factor Four, greenhouse gases, methodology, technology Economy models

Mots-clés : émissions, facteur 4 , gaz à effet de serre, méthodologie, mix énergétique, modèles technico-économiques, politiques climatiques, prix du carbone, scénarios énergétiques

\section{AUTEURS}

\section{SANDRINE MATHY}

Sandrine Mathy est Chargée de Recherche CNRS au CIRED (Centre International de Recherche sur l'Environnement et le Développement). Ses axes de recherches s'organisent autour de la recherche de synergies entre environnement (politiques climatiques) et développement, les régimes climatiques et la modélisation technico-économique sur la prospective énergétique et l'évaluation des politiques de réduction des émissions de gaz à effet de serre. CIRED, 45, avenue de la belle Gabrielle, 94736 Nogent-sur-Marne cedex France Tel : +33 14394 73 96. mathy [at]centre-cired.fr

\section{MEIKE FINK}

Meike Fink est chargée de mission climat \& énergie au Réseau Action Climat - France. Elle est coordinatrice d'un projet européen de recherche "ENCI-Lowcarb" qui vise à développer des scenarios énergétique ambitieux et socialement acceptables pour la France et l'Allemagne. Elle travaille également sur des scénarios à l'échelle infranationale. Avant de joindre l'équipe du Réseau Action Climat, elle était Conseillère Info énergie à l'Idemu, Paris. Son diplôme : Maîtrise en sciences politiques, sciences environnementales et en économie d'entreprise. Réseau Action Climat - France (RAC-F), 2B rue Jules Ferry - 93100 Montreuil France - Tel : +3314858 8979 meike[at]rac-f.org

\section{RUBEN BIBAS}

Ruben Bibas est chercheur au CIRED (Centre International de Recherche sur l'Environnement et le Développement). Il travaille sur des outils et méthodes de prospective de long terme : modélisation énergie-économie-environnement, développement du modèle IMACLIM-R (niveau national et international), élaborations de scénarios de long-terme. Ses thèmes de recherche incluent les politiques de réductions des émissions de gaz à effet de serre : règles de coordination internationale, régimes post-Kyoto, choix d'instruments, évaluation des coûts et des potentiels de réduction. CIRED, 45, avenue de la belle Gabrielle, 94736 Nogent-sur-Marne cedex France Tel : +33143947386; ruben.bibas[at]centre-cired.fr 